\title{
Self-healing UI: Mechanically and Electrically Self-healing Materials for Sensing and Actuation Interfaces
}

\author{
Koya Narumi ${ }^{12 *}$, Fang Qin ${ }^{3 *}$, Siyuan Liu ${ }^{4}$, Huai-Yu Cheng ${ }^{4}$, Jianzhe Gu${ }^{1}$, \\ Yoshihiro Kawahara ${ }^{2}$, Mohammad Islam ${ }^{4}$, Lining Yao ${ }^{1}$ \\ ${ }^{1}$ Human-Computer Interaction Institute, Carnegie Mellon University \\ ${ }^{2}$ The University of Tokyo ${ }^{3}$ Electrical and Computing Engineering, Carnegie Mellon University \\ ${ }^{4}$ Materials Science and Engineering, Carnegie Mellon University \\ \{narumi, kawahara\}@akg.t.u-tokyo.ac.jp, \{fangq, sliu2, hpc, jianzheg, liningy\}@ andrew.cmu.edu, \\ mohammad@cmu.edu
}

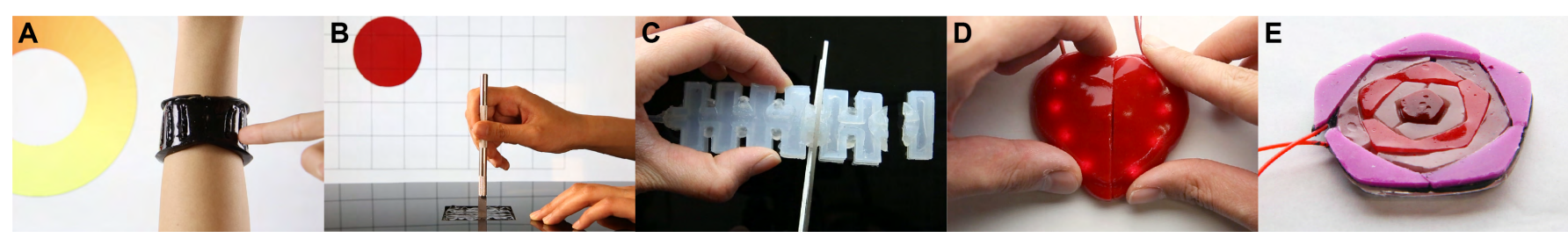

Figure 1. Application examples. (A) A transformative soft controller. (B) A Conformable damage sensor matrix. (C) A reconfigurable pneumatic actuators. (D) A self-healing heart with embedded LEDs. (E) A fusible, color-changing rose puzzle.

\begin{abstract}
Living things in nature have long been utilizing the ability to "heal" their wounds on the soft bodies to survive in the outer environment. In order to impart this self-healing property to our daily life interface, we propose Self-healing UI, a soft-bodied interface that can intrinsically self-heal damages without external stimuli or glue. The key material to achieving Self-healing UI is MWCNTs-PBS, a composite material of a self-healing polymer polyborosiloxane (PBS) and a filler material multi-walled carbon nanotubes (MWCNTs), which retains mechanical and electrical self-healability. We developed a hybrid model that combines PBS, MWCNTs-PBS, and other common soft materials including fabric and silicone to build interface devices with self-healing, sensing, and actuation capability. These devices were implemented by layer-by-layer stacking fabrication without glue or any post-processing, by leveraging the materials' inherent self-healing property between two layers. We then demonstrated sensing primitives and interactive applications that extend the design space of shape-changing interfaces with their ability to transform, conform, reconfigure, heal, and fuse, which we believe can enrich the toolbox of human-computer interaction (HCI).
\end{abstract}

\footnotetext{
*The first two authors contributed equally to this work.

Permission to make digital or hard copies of all or part of this work for personal or classroom use is granted without fee provided that copies are not made or distributed for profit or commercial advantage and that copies bear this notice and the full citation on the first page. Copyrights for components of this work owned by others than the author(s) must be honored. Abstracting with credit is permitted. To copy otherwise, or republish, to post on servers or to redistribute to lists, requires prior specific permission and/or a fee. Request permissions from permissions@ acm.org.

UIST '19, October 20-23, 2019, New Orleans, LA, USA

(C) 2019 Copyright held by the owner/author(s). Publication rights licensed to ACM. ISBN 978-1-4503-6816-2/19/10.. \$15.00

DOI: https : //doi .org/10 . 1145/3332165. 3347901
}

Note: Carbon nanotubes are categorized into carcinogenic materials that require the proper preparation process by those who took safety training with suitable equipment.

\section{CCS Concepts}

-Human-centered computing $\rightarrow$ Interaction devices; User interface design; User interface toolkits;

\section{Author Keywords}

Self-healing materials; material-driven interfaces; shape-changing interfaces; sensing and actuation system; bio-inspired interfaces; radical atoms; soft matters.

\section{INTRODUCTION}

From lizard tails to starfish arms, from grafted trees to human skin, the regenerative and self-healing phenomena are ubiquitous in nature. Inspired by how natural self-healable systems can restore their mechanical integrity and functionality (e.g., nervous system, nutrient canals) in an autonomous manner, we propose self-healing UI that mimics and exploits this unique healing property to design interface systems around us (e.g., wearables, soft interface, digital fabrication, art).

In human-computer interaction (HCI), shape-changing softbodied interfaces [8, 42, 2], radical atoms [15], deformable interfaces [4], and material-driven interfaces [41] are drawing increasing attention. This is due to the fact that shape change provides unique form, function, and interaction, represented by wearable sensors [17, 34, 52], deformable mobile devices [13], pneumatically actuated daily products [58], devices with haptic feedback [55], and interactive robotics [50]. However, there exist three major challenges when dealing with soft materials: 
(1) soft material systems are fragile; once broken, there is often no easy way to fix them; (2) the fabrication process of soft material systems can be tricky; in particular, construction of an air bladder requires a highly skilled process with a large failure rate; (3) especially in case of interfaces made from silicone rubbers [58], the bonding or connection with other materials is difficult once the silicone has completely cured; (4) conductive soft materials often cannot be connected to bulk metal conductors with conventional soldering techniques, and connections made on soft materials become weak.

In this paper, we present Self-healing UI, a soft-bodied material interface that follows these principles: (1) the devices can self-heal for repeated use; (2) the material affords a novel solution for device fabrication that is able to fuse both conductive and non-conductive components; (3) the material sticks to a variety of soft materials such as fabric and silicone; (4) the interface is able to integrate both input sensing and output actuation. Furthermore, we exploited self-healability of our material to demonstrate a novel design space of shapechanging interfaces. Examples of Self-healing UI for HCI applications are illustrated in Figure 1.

The main contributions of this paper are as follows:

- Proposing a layered model for Self-healing UI, including stiffness-/conductivity- tunable self-healing polymers, as well as other common soft materials such as silicone and fabric.

- Developing a preparation and molding method of PBS and MWCNTs-PBS sheets by extending previous literature [56].

- Building a layer-by-layer stacking fabrication method that can achieve a continuous structure without additional gluing or bonding processes.

- Exemplifying the design space of Self-healing UI by incorporating its transformative, conformable, reconfigurable, healable, and fusible nature in proposed applications.

\section{RELATED WORK}

\section{Self-healing materials}

Basic principle and category studied in materials science

Traditional polymers, which are typically crosslinked through irreversible covalent bonds, have been widely used in the modern industry. However, due to the strong bonding, these polymers lose their functionality after being damaged [46]. Hence, scientists and engineers developed several strategies to allow the system to restore its functionality.

Self-healing polymer is classified into four categories by these two factors, "extrinsic vs. intrinsic" and "autonomous vs. non-autonomous" properties. The self-healing mechanism explored first is extrinsic self-healing, represented by capsules within the polymer matrix $[20,54]$. The damage will crack these small capsules, and the self-healing component will flow out and react to recover the operator area $[20,54]$. However, these self-healing systems are not able to recover from the repeated damage due to the depletion of self-healing materials in the capsules.
In contrast, intrinsic self-healing polymers, with dynamic crosslinking bonds, have been developed to regain their properties repeatedly in different mechanisms [6, 23, 33, 40]. Some of them require non-autonomous external stimuli such as light, heat, and force which allow the self-healing procedure to be controllable [7]. On the contrary, other systems can self-heal autonomously without any external stimuli. Many autonomous self-healing systems are made from hydrogels, which are mostly composed of water or organic solvents and therefore are widely used in biomedical materials [44]. However, they tend to involve degradation caused by evaporation of solvent [25, 24], which is not suitable for daily life application.

Thus, in this paper, we focused on polyborosiloxane (PBS) as a candidate material for $\mathrm{HCI}$, which retains intrinsic and autonomous self-healing property. It is also biocompatible and solvent-free, which leads to easy, safe fabrication and use and longer material lifetime [19, 25, 24]. Multi-walled carbon nanotube (MWCNTs) were also introduced as the filler to impart conductivity to the polymer. Both PBS and MWCNTsPBS are biocompatible under appropriate use (see Material Safety section for more discussion), and is suitable for daily life interface. Although conductive self-healing composites and flex sensors utilizing PBS were previously reported [11, 56], their discussion was mainly limited to a few physical properties, and interface systems with a hybrid structure of conductor, insulator, and substrates have not been explored.

\section{Existing applications of self-healing phenomena}

Although it is still not pervasive, self-healing properties of materials are applied in several fields. As commercialized examples, we have access to self-healing coating for automobiles and displays. Self-healing concrete [16] for sustainable architecture and infrastructure is also readily available on the market. In the field of robotics, self-healing property of liquid (e.g., oil, liquid metal) has been explored for soft actuators [1, $18,47]$ and robust robots [28]. The most related example of self-healing phenomenon to digital fabrication or HCI might be fused deposition modeling (FDM) for 3D printing, in which thermoplastic fuses and sticks to the previous layer to build a continuous 3D object.

\section{Shape-changing interfaces}

Shape-changing interfaces have already been intensively explored and reviewed $[8,42,2]$. Here, we classify previous studies about shape-changing interfaces by three phases of materials: solid, liquid, and solid-liquid, and explain the main types of shape change each phase affords.

\section{Solid shape-changing interfaces}

Solid shape-changing interfaces utilize dynamic mechanisms (e.g., hinges, pistons) or the relative position of many stiff objects, which result in precise, fast, and reproducible motion. Above all, many researchers have recently focused on the latter strategy such as a 1D line and chain [31,30], 2D pin displays [12,32] and swarms [21], and dynamic 3D printing using magnetic blocks [45]. These examples all exploit the reconfigurable nature of physical elastic pixels or voxels. 


\section{Liquid shape-changing interfaces}

Liquid shape-changing interfaces, on the other hand, mainly utilize the healing and fusing nature of two isolated portions of liquid into a single droplet. For example, Programmable blobs [49] uses magnetic fluid to demonstrate a liquid display that changes its shape depending on the magnetic field. LIME [26], Tangible Drops [43], and Programmable Droplets [48] controls the shape, number, and position of liquid metal or aqueous solutions by an electric field. Despite its high degree of freedom in shape and potential for fusing interface, they are still hard to handle due to the viscose, fluidic nature.

\section{Solid-liquid shape-changing interfaces}

Solid-liquid materials [25] represented by silicone rubbers and dough retains viscoelastic property; by tuning their property, they can sustain its shape, while deforming dynamically. HCI researchers have developed many soft-bodied interfaces using these materials, such as pneumatic actuation interface [14, $37,58]$, flexible, stretchable electronics [36, 53], and dough interfaces such as a conductive tack [38], sand [51], or a toy called Silly Puty [5]. They are able to transform and conform (while keeping their shapes) by the solid-liquid property of materials.

Our proposing Self-healing UI also falls into the solid-liquid shape-changing interface: the key difference to conventional solid-liquid interfaces is that our UI can heal and fuse like liquid, while achieving reconfigurability, transformability, and conformability that solid and solid-liquid interfaces offer. We will demonstrate each property by our proposing applications.

\section{DESIGN CHOICES OF MATEIRALS FOR HCI}

As explained in Related Work, we used PBS [19, 25, 24] as a main self-healing material candidate for HCI for the following reasons:

1. Intrinsic and autonomous self-healing ability without any external stimuli (e.g., heat, light, additive materials causing chemical reactions) to avoid complex environmental setup.

2. Controllable electrical and mechanical properties depending on the applications. The property of PBS can be tuned by dispersing varying amounts of MWCNTs into the polymer to form MWCNTs-PBS composites.

3. Robust material for long-term use in a daily environment without time-dependent degradation.

4. Simple and low-cost fabrication with which researchers in the HCI field can leverage after training.

5. Safety and compatibility to human skin and conventional substrate (e.g., acrylic, polydimethylsiloxane (PDMS), fabric).

Figure 2 shows that two separated PBS samples can quickly self-heal and recover its mechanical strength. In terms of its working mechanism, PBS is a room-temperature self-healing supramolecular polymer that is crosslinked through the dynamic bonds between boron and oxygen [19, 25, 24] (Figure 3). The boron atom in one PBS chain can form a dynamic bond

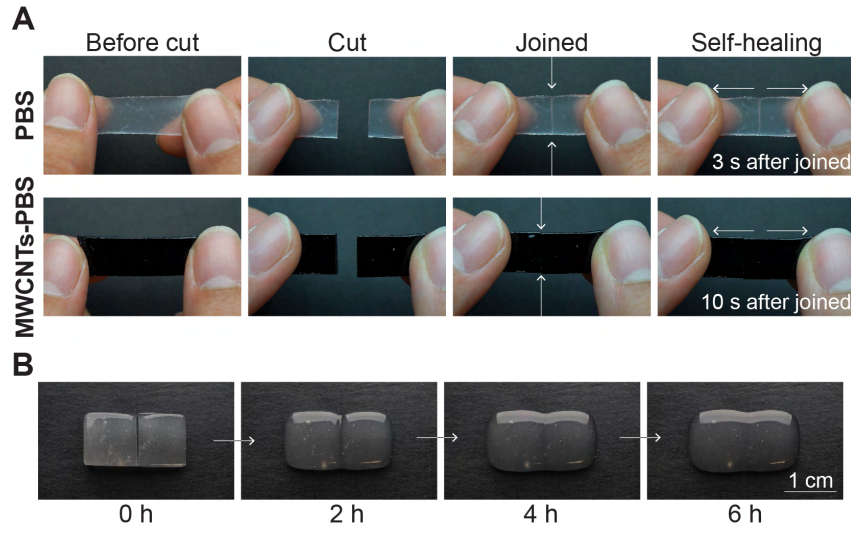

Figure 2. Self-healing process of PBS and MWCNTs-PBS. (A) The rapid healing process of PBS and MWCNTs-PBS. (B) The long-term healing process of PBS, where we observed the cut lines of samples disappearing over time.

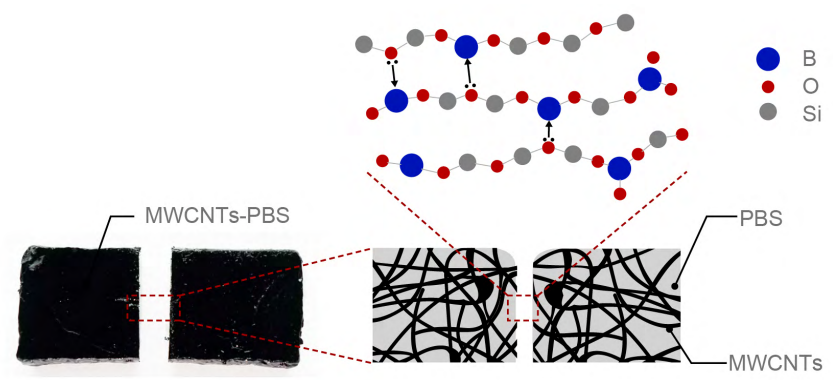

Figure 3. The working mechanism of MWCNTs-PBS. The dynamic bonds between boron and oxygen cause self-healing of the PBS matrix.

with the oxygen atom from a nearby chain. However, PBS has a weak mechanical strength [19, 25, 24] and low conductivity. Therefore, MWCNTs was suggested as fillers that compose networks to tune its mechanical and electrical performance [54]; in the latter sections, we report performance evaluations of PBS and MWCNTs-PBS developed in our method.

\section{PREPARATION OF MATERIALS}

In this section, we describe the preparation of PBS and MWCNTs-PBS. We note that MWCNTs are categorized into carcinogenic materials, we need to prepare the safety equipment and follow the procedure written here. All the preparation processes were done inside the fume hood along with appropriate disposal for wastes containing MWCNTs. Also, throughout the preparation, we wore disposable gloves and masks to avoid the direct contact and inhalation of MWCNTs. For more information on safety, see the latter section for material safety.

We have adopted conventional protocols from the literature [56] with additionally tailored procedures (sonication, doctor blading, and dilution explained below). Both PBS and MWCNTs-PBS were prepared on three criteria: large quantity preparation, the form factor of $2 \mathrm{D}$ thin sheets needed to stack them into 3D devices, and easy-to-follow material handling procedures, in order to fit in our layer-by-layer stacking fabrication strategy described later. 
Preparation of PBS sheets

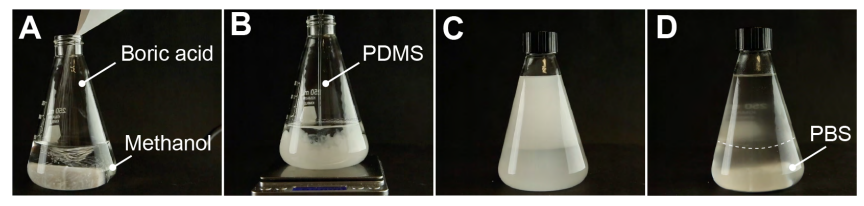

Figure 4. Preparation process of PBS.

The boric acid-methanol solution was prepared by adding 1.0 $\mathrm{g}$ of boric acid (B6768; Sigma) into $10 \mathrm{~mL}$ of methanol and continuously mixed for $1 \mathrm{~h}$ with a magnetic stir (Figure 4A). Then $10 \mathrm{~g}$ of PDMS (silanol terminated, 35-45 cSt; Gelest) was added to the boric acid-methanol solution and vigorously mixed together for $3 \mathrm{~h}$ (Figure 4B,C). After that, the mixed solution was placed on a static table for $1 \mathrm{~h}$ until two separate liquid phases are clearly observed (Figure 4D). Uncured PBS was extracted from the bottom part using a transfer pipet. At this point, a small portion of silicone pigment (Silc-Pig; Smooth-on) can optionally be mixed with the solution. This solution was poured into a laser-cut acrylic mold coated with mold release spray (Food Grade Mold Release W0, CRC). The uncured PBS solution in the mold was then placed inside an oven at $60{ }^{\circ} \mathrm{C}$ for $24 \mathrm{~h}$ to fully cure.

\section{Preparation of MWCNTs-PBS sheets}

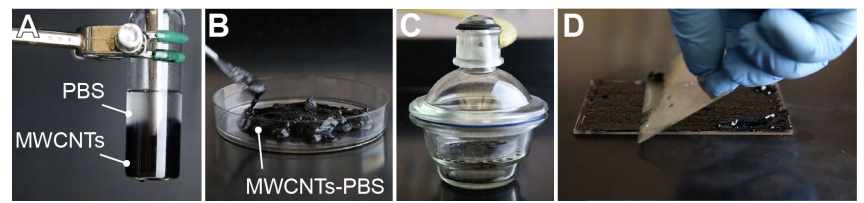

Figure 5. The preparation process of MWCNTs-PBS sheets.

MWCNTs (Baytubes C $150 \mathrm{HP}$ ) were added into the uncured PBS solution and sonicated (Fisher Scientific Sonic Dismembrator Model 500) at an amplitude of $100 \mathrm{~W}$ for $1 \mathrm{~h}$ to debundle the MWCNTs (Figure 5A,B). The dispersion was vacuumed to remove extra methanol (Figure $5 \mathrm{C}$ ), poured into a mold, and transferred into an oven at $60^{\circ} \mathrm{C}$ for $24 \mathrm{~h}$ to allow the sample to fully cure. Once cured, the sample becomes safe to use and cut with bare hands. During the molding process, we spread the surface of the MWCNTs evenly with a straight knife (Figure 5D, so-called doctor blading) to get a smooth shape of sheets. We newly adopted sonication process to disperse MWCNTs more homogeneously than the conventional method [56], which led to higher conductivity of the material with a lower concentration of MWCNTs in PBS.

We note that uncured PBS can be stored in room temperature for over 1 month, while uncured MWCNTs-PBS dispersion must be used right after sonication to prevent aggregation and bundling of MWCNTs. If we use an old uncured MWCNTsPBS dispersion sample, the sample needs a sonication process again. In addition, in order to prepare materials in large quantity and with precise MWCNTs fraction, we adjusted the conventional procedure by starting with the preparation of high-concentration MWCNTs-PBS and then subsequently dilute it with additional uncured PBS solution.

\section{PERFORMANCE EVALUATION}

We have conducted a detailed performance evaluation for both electrical and mechanical properties of PBS and MWCNTsPBS, both before and after cut and joined. The data shown in this section are from 5 independent samples and measurements. The error bars shown are the maximum/minimum values of the samples. All the other plots show the mean value for each condition. The sample size was L: $12.5 \mathrm{~mm}, \mathrm{~W}: 6.10 \mathrm{~mm}, \mathrm{H}$ : $1.32 \mathrm{~mm}$ on average. For electrical measurement, we covered both sides of each sample with silver epoxy (CP4922N-100; DuPont) and applied 2 probes to the silver parts to measure resistance. For mechanical measurement, we used a load measurement machine (Instron 5940).

\section{Electrical property of MWCNTs-PBS}
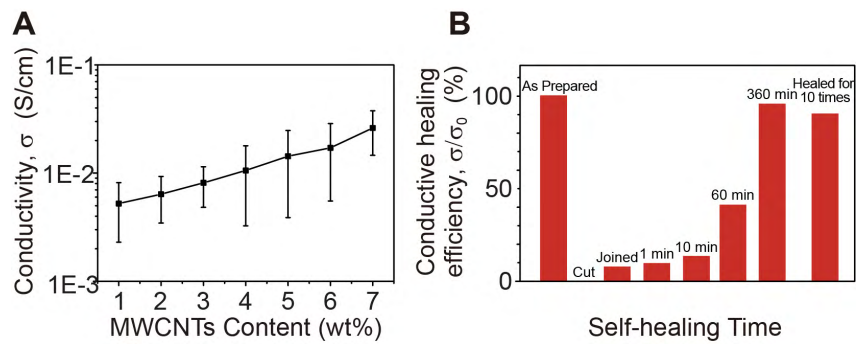

Figure 6. The electrical property of PBS and MWCNTs-PBS. (A) Conductivity vs weight fraction of MWCNTs in the samples. (B) Conductive healing efficiency depicting the time-dependent electrical self-healing property.

In order to determine the suitable composition of MWCNTsPBS for our devices, we measured electrical conductivity as a function of MWCNTs content. Figure 6A shows the conductivity of MWCNTs-PBS with different fractions of MWCNTs. Although the error bars overlap, we observed that the mean electrical conductivity showed a clear upward tendency with increasing MWCNTs content. The resultant conductivity was more than 10 times higher than the previous report when comparing $3 \mathrm{wt} \%$ samples [56]. This higher conductivity can be attributed to the homogeneous network of MWCNTs inside PBS by the sonication process instead of mechanical stir [56]. To meet the electrical conductivity requirement for the devices, we used the MWCNTs content of $4 \mathrm{wt} \%$ for all the other experiments and applications in this paper. We note that pure polymers like polydimethylsiloxane (PDMS), which is similar to PBS, have a conductivity ranging between $10^{-16}-10^{-12}$ $\mathrm{S} / \mathrm{cm}$ [27].

In addition, to investigate the electrical self-healing property, we cut and joined the composite. Conductive healing efficiency, defined as $\sigma / \sigma_{0}$, at different healing time was plotted in Figure 6B, where $\sigma_{0}$ is the electrical conductivity of the as-prepared samples and $\sigma$ is the electrical conductivity at different self-healing times. We found that $10 \%$ of the electrical conductivity immediately recovered after we joined the two halves. Then, the electrical conductivity slowly recovered over time. After $6 \mathrm{~h}, 96 \%$ of electrical conductivity was recovered. We also tested electrical conductivity for the samples after 10 damaging-healing cycles to verify the repeated self-healing property. As shown in Figure 6B, approx. 90\% of the electrical conductivity recovered after 10 damaging-healing cycles. 


\section{Mechanical property of PBS and MWCNTs-PBS}

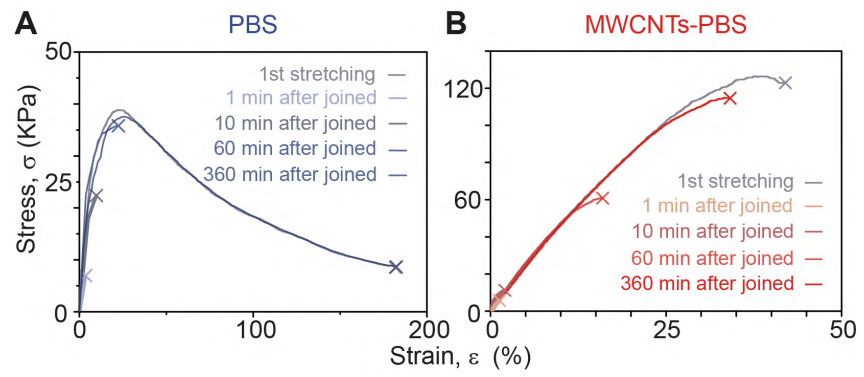

Figure 7. The mechanical property of (A) PBS and (B) MWCNTs-PBS. " $\times$ " mark means the sample at break.

To investigate the mechanical self-healing properties, we tested the tensile stress $\sigma$ vs. strain $\varepsilon$ over the range of $\varepsilon=0-200 \%$. The mechanical properties of PBS and MWCNTs-PBS are shown in Figure 7A,B, respectively.

PBS showed the elastic region for $\varepsilon<5 \%$. The sample reached the ultimate stress of $39 \mathrm{kPa}$ at $\varepsilon=39 \%$, but is able to be stretched to $\varepsilon=180 \%$ before a fracture occurred. To study mechanical self-healing, we cut the sample into halves and then joined them. We allowed the samples to self-heal for a certain amount of time before measuring the curve. As shown in Figure 7A, the ultimate elongation strain of PBS increased with more self-healing time, and after $6 \mathrm{~h}$ the samples exhibited the almost identical tendency as as-prepared samples.

The same measurements were performed on MWCNTsPBS with $4 \mathrm{wt} \%$ MWCNTs content. The elastic region of MWCNTs-PBS was extended to $\varepsilon=8 \%$. Moreover, adding the MWCNTs filler makes the composite stronger, whose ultimate stress was enhanced to $120 \mathrm{kPa}$ at strain $\varepsilon=40 \%$ as shown in Figure 7B. On the other hand, the addition of MWCNTs fillers slowed down the self-healing speed. As shown in Figure 7B, the composite did not fully restore its original mechanical property after $6 \mathrm{~h}$.

\section{SELF-HEALING UI AS A HYBRID MATERIAL SYSTEM}

As we evaluated in the previous section, PBS and MWCNTsPBS have pros and cons. Here, we propose a novel hybrid design that takes a middle way. We leveraged the fast healing and electrically insulating property of PBS, as well as the electrical conductivity and mechanical stiffness of MWCNTsPBS to implement practical interface devices.

\section{Balance between PBS and MWCNTs-PBS}

Balance in self-healing speed. As explained in Figure 7, the self-healing speed of MWCNTs-PBS is slower despite its conductive property, while insulative PBS can heal faster. Thus, by combining PBS and MWCNTs-PBS, we can achieve fast mechanical healing while keeping conductivity.

Balance in stiffness. PBS changes its shape as time passes due to gravity (so-called a "creeping" effect shown in Figure 8), which is problematic for everyday use from the viewpoint of users. On the other hand, MWCNTs-PBS can hold its shape thanks to its sustainable network structure as shown in Figure 3. Thus, by exploiting MWCNTs-PBS as a substrate of PBS, the whole system can maintain its shape for more robust usage.

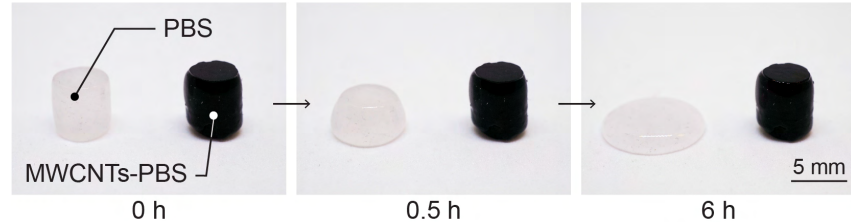

Figure 8. Creeping effect of PBS.

\section{Functions of each layer}

Figure 9 shows an overview of our layer-by-layer stacking model. The whole structure is composed of three different types of layers: a substrate layer, one or multiple functional healing layers, and a covering layer.

The substrate layer. It works as elastic mechanical support (e.g., Ecoflex 00-30 silicone (Smooth-on), PDMS (Sylgard 184; Dow Corning), and MWCNTs-PBS shown in Figure $10 \mathrm{~A}, \mathrm{~B}, \mathrm{C})$ that restricts PBS layers from flowing and creeping over time. We note that PDMS and many other silicone elastomers are commonly used for HCI applications [36]. On the other hand, a composite of PBS and polyester fabric provides extra adhesion to the skin (Figure 10D). To achieve these substrates bonded with the functional layers, we poured uncured PBS solution on the cured PDMS and Ecoflex and put them in $60{ }^{\circ} \mathrm{C}$ oven for $24 \mathrm{~h}$. MWCNTs-PBS was just joined together with PBS, and they fused into the continuous structure with their inherent self-healability. We made PBS-polyester fabric by first soaking fabric into uncured PBS solution, which was then vacuumed for approximately $30 \mathrm{~min}$ until no air bubbles were observed. This fabric composite was fully cured at $60^{\circ} \mathrm{C}$ for $24 \mathrm{~h}$.
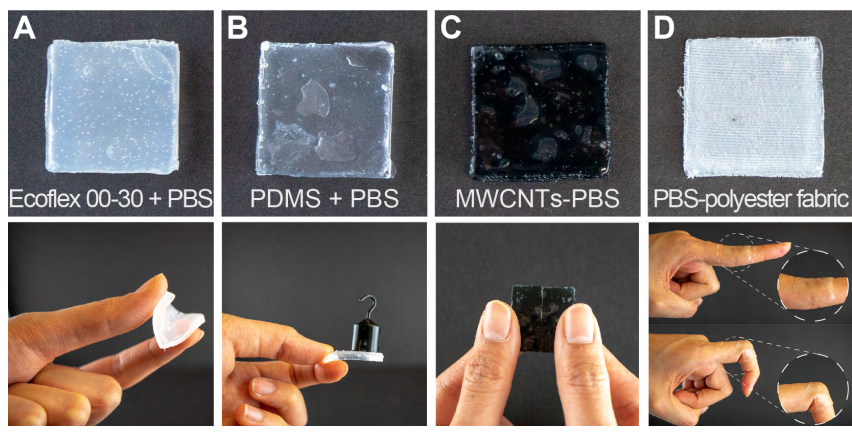

Figure 10. Options for the substrate layer. (A) Commercially available soft silicone (Ecoflex 00-30). (B) PDMS as a stiff substrate to hold the shape. (C) MWCNTs-PBS that better sustains the shape than pure PBS while achieving self-healability of the whole structure. (D) PBS-polyester fabric sticking and conforming to human skin.

The functional layer. It is composed of PBS, MWCNTsPBS, miniaturized electronic components, connection wiring to the outer module, and some other functional layers (e.g., color changing composites). MWCNTs-PBS can work as sensors for pressure, touch, and cut. PBS in this layer has three major functions: effective self-healing medium, insulation components between conductive MWCNTs-PBS and other electronics, and integration matrix to embed other wires and components into one continuous body. 


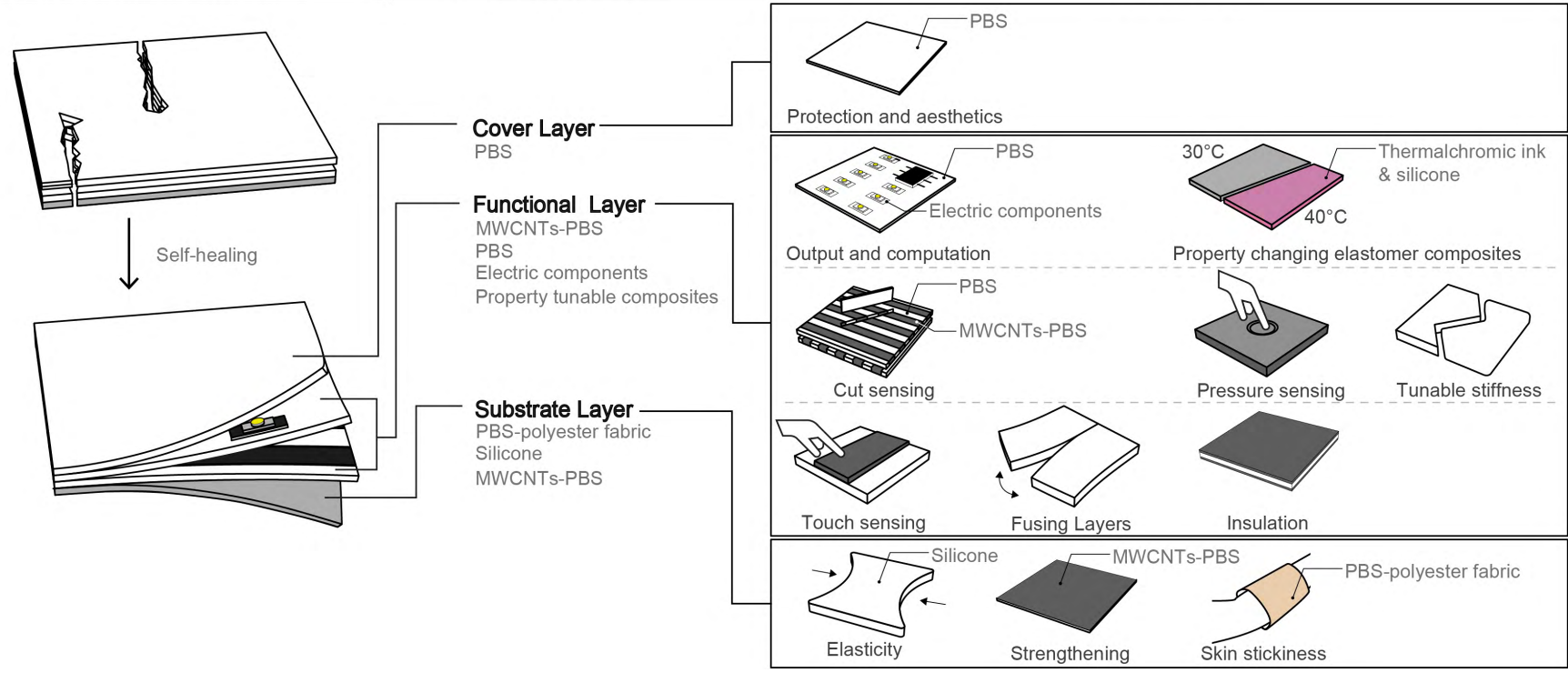

Figure 9. A layered Model of Self-healing UI. The substrate layer sustains the whole device to keep its shape and works as an interface to other materials or the human body. The functional layers work as a self-healing body, circuity, sensors, or other functional media (e.g., the color changing layer). The cover layer is optionally used for protection of the device and aesthetics.

The covering layer. This layer is optionally used for the sake of protection, insulation, and aesthetics.

\section{LAYER-BY-LAYER STACKING FABRICATION}

Leveraging the self-healability of PBS, our fabrication process exhibits four advantages over conventional layer-stacking techniques: no need for adhesives between layers; no need for soldering between conductive wires and MWCNTs-PBS; the flexibility to stabilize electronic components between layers; the stable bonds between PBS and other silicone elastomers. In this section, we describe the fabrication process of Self-healing UI by stacking PBS, MWCNTs-PBS, and other components.

\section{Step 1: fabrication of each layer}
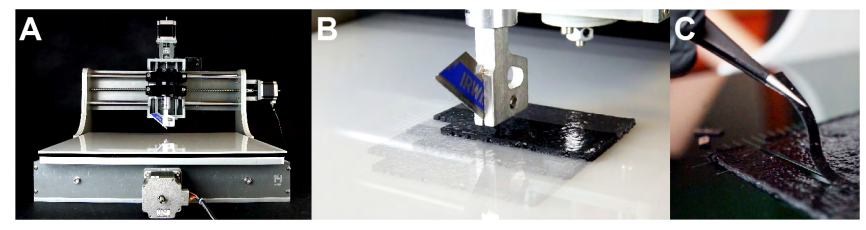

Figure 11. Cutting PBS and MWCNTs-PBS. (A) the materials were removed from the mold and cut by (B) a CNC drag knife or a die cutter into designated components. (C) Then each component was placed in order, and the whole structure automatically self-heals and fuses.

First, PBS and MWCNTs-PBS sheets were removed from the molds carefully with tweezers. Then each material was cut by a CNC drag knife (12'x12' CNC Machine; Zen Toolworks, D2 drag knife; Donek) as shown in Figure 11 or a die cutter (made by laser cutting acrylic boards). After cutting each component, they were placed in order and fused together by their self-healing property.

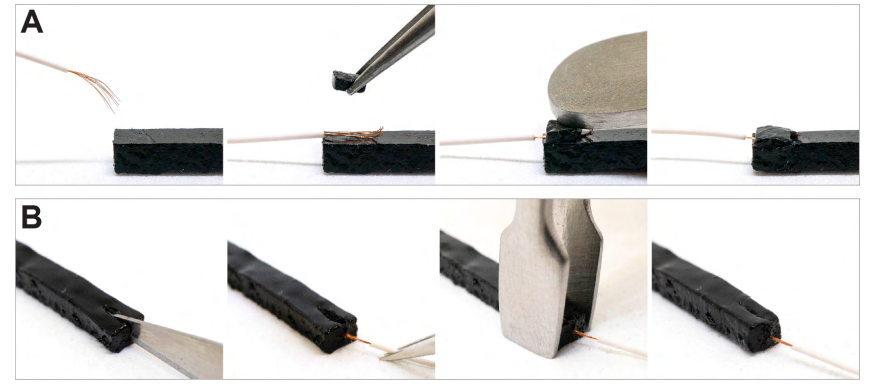

Figure 12. Two wiring methods for MWCNTs-PBS circuitry. (A) Placing additional MWCNTs-PBS piece with tweezers. (B) Cutting the middle part of the trace and insert the wire.

\section{Step 2: electrical connection}

As shown in Figure 12, sandwiching the wire with self-healing material can work both for electrical and mechanical connection without glue. We demonstrated two methods of (1) placing additional MWCNTs-PBS material onto the wire and (2) cutting the middle part of MWCNTs-PBS and inserting the wire inside the gap. The former is easier for a smaller size of MWCNTs-PBS circuitry, while the latter is better in terms of aesthetics. We used multi-strand wire (36 AWG, UAA3607; Micron Meters), for maximizing the surface contact area on MWCNTs-PBS to stabilize the conductivity and minimizing the volume of the connection part to help MWCNTs-PBS self-heal faster.

\section{Step 3: stacking layers}

Once preparation of each layer and connection was done, Each layer and component was stacked in order as shown in Figure 13. We used a laser-cut acrylic board shown in Figure 13B to guide the position of MWCNTs-PBS strips. 


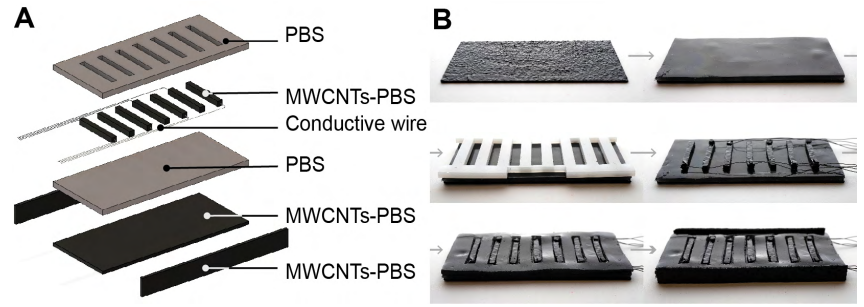

Figure 13. (A) A layered model of a transformative soft controller. (B) Stacking layers in sequence. The device will self-heal and form one continuous body within $6 \mathrm{~h}$. The white acrylic plate was used to guide the position of MWCNTs-PBS strips.

\section{SENSING PRIMITIVES}

In this section we demonstrate three sensing primitives: touch sensing, pressure sensing, and cut sensing. Figure 14 and Figure 15 show the circuit diagram and the photos for each sensing method, respectively. We note that pressure sensing and cut sensing were performed with the same circuit diagram (Figure 14B). For implementation, we used Arduino Mega $2560 \operatorname{Rev} 3$ as a microcontroller.

\section{Touch sensing}

Touch sensing was implemented in the same way as a conventional touch sensor made of a bulk metal conductor. Depending on the distance between the human body and MWCNTsPBS, the capacitance value (shown with a dashed line in Figure 14A) changes, resulting in the configuration of the low pass filter. We used the Capacitive Sensing Library of Arduino.

\section{Pressure and cut sensing}

Each end of MWCNTs-PBS was connected to $\mathrm{V}_{\mathrm{cc}}(=5 \mathrm{~V})$ and a digital pin reading the voltage value. When we apply pressure to the MWCNTs-PBS, it deforms to increase its resistance value. This change in resistance can be read by the digital pin as a decreased voltage. The same mechanism as pressure sensing can be applied to cut sensing. If MWCNTs-PBS is cut into pieces, the voltage value read from the digital pin goes to $0 \mathrm{~V}$, which is clearly detectable. Although this sensing mechanism is as simple as a tact switch, the self-healing property of MWCNTs-PBS allows detecting cuts repeatedly if we make a mechanical connection of each piece, which is a unique property for this sensing method.

A

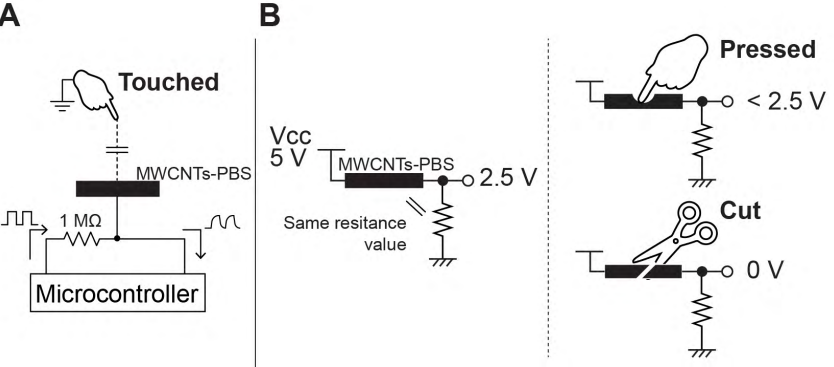

Figure 14. The sensing principle for (A) a touch sensor and (B) a pressure and cut sensor.

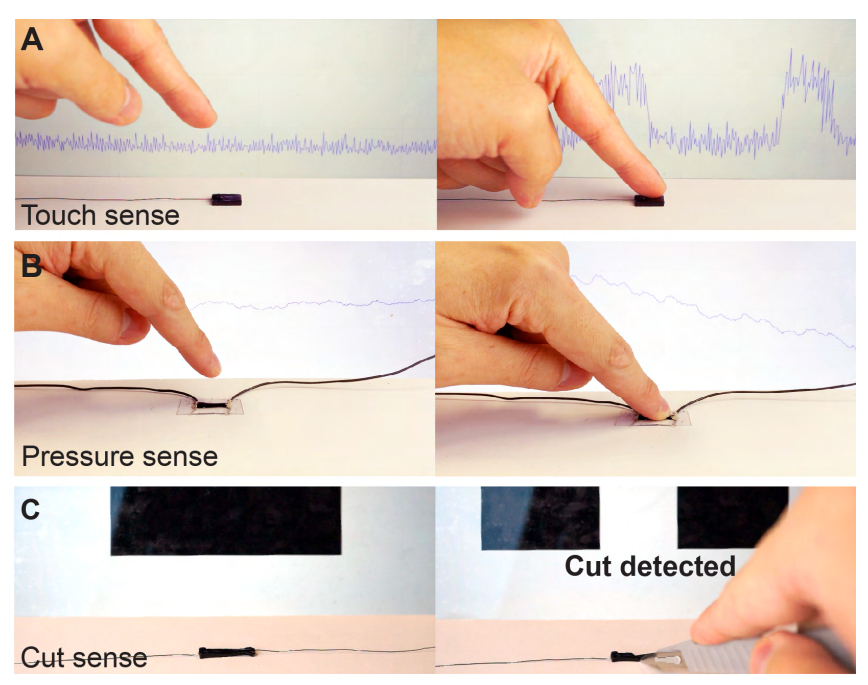

Figure 15. Sensing primitives. touch, pressure, and cut sensors are activated in front of the screen with their input values.

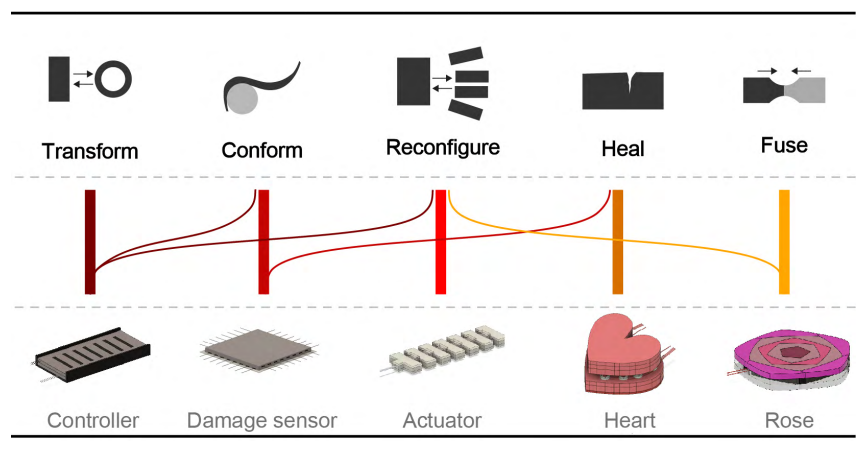

Figure 16. The design space of Self-healing UI.

\section{APPLICATIONS}

In this section, we explored the design space of the Selfhealing UI through five demonstration artifacts. As shown in Figure 16, we can distinguish the functionality of soft, selfhealing materials into five: (1) dynamic transformation of shape; (2) Conformable applications of UIs to other objects; (3) Reconfiguration of the number and the function of devices; (4) Healability of the damaged or broken part; and (5) fusing nature of two different modules. Each of these five spaces is mainly related to five applications we will demonstrate (thick line in Figure 16), but some of them have overlapping features depicted as a thin curved line in Figure 16.

\section{Transformative soft controller}

We envisioned a controller device that can dynamically change its shape and number of modules depending on different ways we expect to it. Figure 12 shows the layered structure of the proposed controller; one of the MWCNTs-PBS strips is a pressure/cut sensor and all the other strips are touch sensors. Wiring was connected to Arduino. A series of touch sensors were used to detect both a single touch and the sliding motion.

Figure 17 shows four use scenarios of this controller. First, a single controller detects a finger press motion in Figure 17A (the normal mode). Then two controllers were joined to form 
long touch buttons and worked as a piano keyboard in Figure 17B (the long mode). Next, two connected controllers wrapped around the user's wrist, working as a wrist band slider device in Figure 17C (the wrist band mode). When three friends came to play a video game, the controller was cut into four pieces and worked as half-sized gaming devices with three touch sensors for each in Figure 17D (the cut mode). After use, the four pieces can be joined into the original two controllers for $6 \mathrm{~h}$.

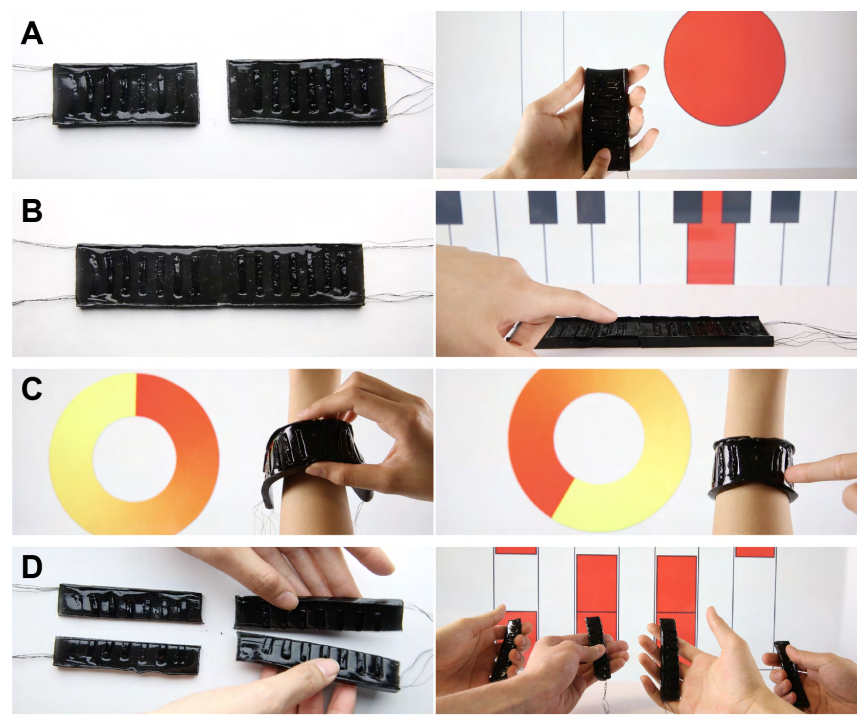

Figure 17. The transformative soft controller can change its shape and reconfigure the number and the function of the device as user needs.

\section{Conformable damage sensor}

We prototyped a self-healing damage sensor matrix as a second skin. The layered model of the damage sensor is shown in Figure 18A,B. The first (substrate) layer was made by PBSpolyester fabric which can stick to human skin (Figure 18C), In the second and the fourth layer, MWCNTs-PBS cut sensors were distributed in series, composing an $8 \times 8$ cut sensor matrix. The third PBS layer was added for insulation between two conductive layers.

Figure 19A shows a demonstration of how it works. First, when we made a single cut at one node of the matrix, the red dot showed up on the screen and visualized the damaged point. Next, we made another cut on the damage sensor matrix, and then the red circle on the screen got bigger, showing the region of two cuts. We again cut the third node, and the circle got bigger again. Figure 19B shows almost the same situation, but in this case, three cuts are on the same line. Then the screen showed a more precise position of damages. We note that the damages in Figure 19A were expressed as a big circle, not multiple points, because we suffered from a "ghosting effect" of the sensor matrix, which will be discussed later.

\section{Reconfigurable pneumatic soft actuator}

Conventional pneumatic soft actuators were subject to the small damage; we propose a cuttable and thereby reconfigurable version of such actuators with PBS. Figure 20 shows the layered model and the photo of the proposed pneumatic
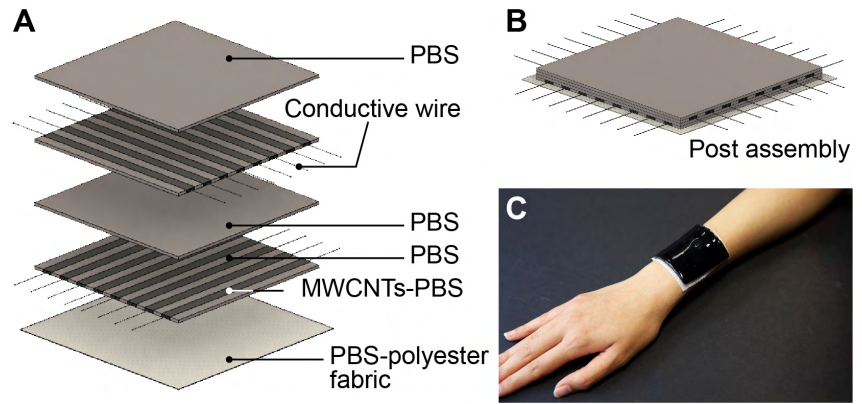

Figure 18. (A, B) A layered model of $8 \times 8$ damage sensor pad. (C) The soft sensor pad sticks and conforms to skin. This photo was taken without wiring to the sensor for clarity.

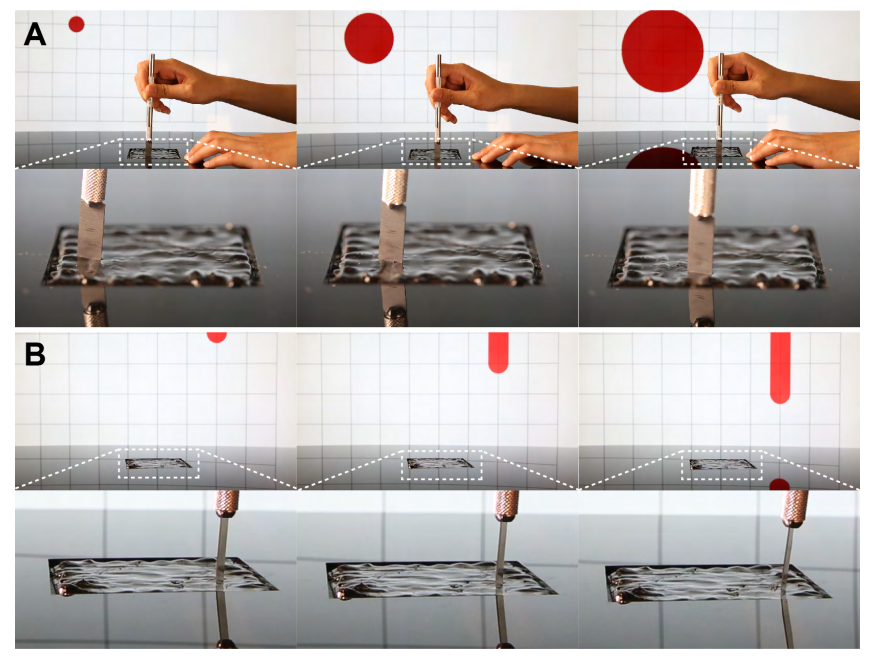

Figure 19. Demonstration of the damage sensor matrix on the table. (A) Three diagonal cuts induced a damaged area bigger in sequence. (B) Three linear cuts made the red line on the screen longer, implying the cut gets longer.

actuator. The top and the bottom parts of the pneumatic channel were made from two different silicone Ecoflex 00-30 and Dragon Skin 00-30 (Smooth-On) with different stiffness, which led to anisotropic bending motion when we supply air. We coated each node of the actuator with PBS, which keeps the pneumatic channel airtight ever after cut and joined.

Originally, the actuator bent into a " $\mathrm{C}$ " curve (Figure 21A). After cutting one node of the actuator and attaching the cut piece upside down, however, the actuator formed an "S" curve (Figure 21B). When we shortened the length of the actuator and attached the end piece back, a shorter version of the "C" curve still worked in the same way as the longer one (Figure 21C).

\section{Healing heart}

We mimicked the heart warming/breaking moment with our self-healing materials. Figure 22 shows the layered model of the self-healing heart and its assembling procedure. We embedded LED components and MWCNTs-PBS cut sensor inside. When the cut sensor detects the mechanical and electrical connection, LEDs are lit up as feedback. The actual workflow is shown in Figure 23. First, two users joined the heart, and the LEDs reacted to the physical and mental connection 


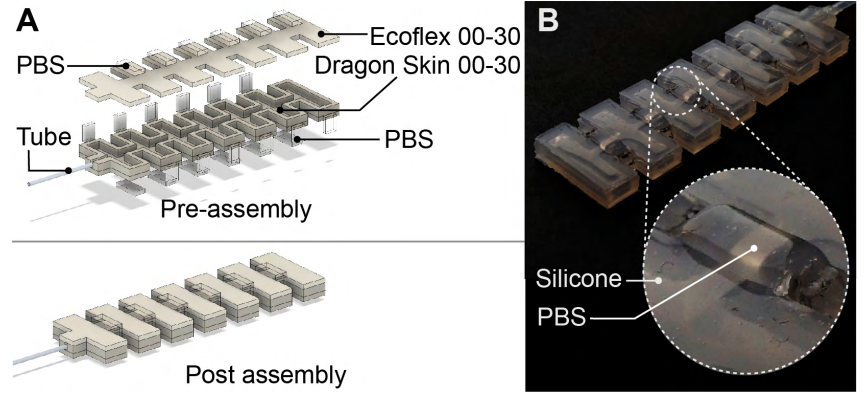

Figure 20. (A) A layered model of the reconfigurable soft actuator. (B) PBS wraps around and covers each node.
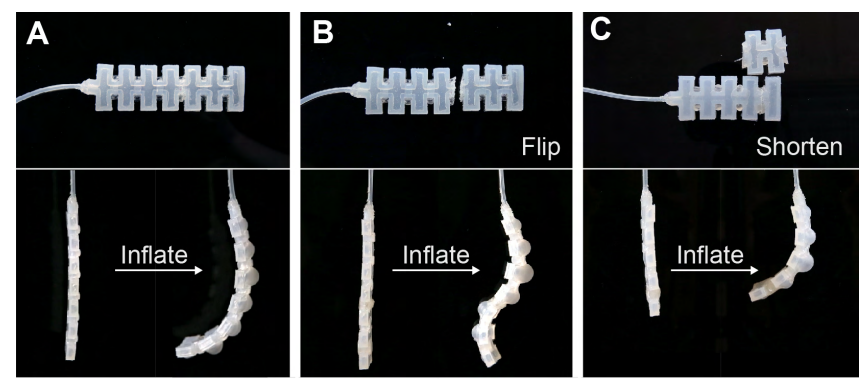

Figure 21. The photos of different configurations and functions of the actuator.

(Figure 23A,B). Over time, the gap between the two pieces gradually got vague and disappeared in $6 \mathrm{~h}$ (Figure 23C). Finally, the shape of the heart became continuous in Figure 23D.

\section{Fusing modular rose puzzle}

We utilized the fusing property of different modules, and made a puzzle with a rose shape. As shown in Figure 24A, it is basically composed of PBS pieces with different colors, but only the outer pieces are made of Ecoflex mixed with thermochromic ink, MWCNTs-PBS conductor, and PDMS. This outer layer works both as (1) a mechanical constraint which prohibits the inner PBS pieces from flowing out and (2) an electrical heater with logic. When users finished the assembly shown in Figure 24B-F, MWCNTs-PBS in the outer pieces were electrically connected and heated up by Joule heat, which turned the black color of the thermochromic layer into pink (Figure 25A,B). Moreover, as time passed, each piece fused into one smooth and continuous structure as shown in Figure 25C,D.

\section{MATERIAL SAFETY IN PREPARATION AND USE}

We put this discussion section in order to inform of appropriate knowledge on danger our materials potentially have. In summary, the use of Self-healing UI is skin-compatible in the room temperature, and users can handle and cut it with bare hands as demonstrated below. However, the preparation process clearly involves manipulation of dangerous substances and this should be done by those who took the proper training under the suitable environment.

\section{Safety of MWCNTs}

The main safety concern for MWCNTs use is inhalation, which may bring them in contact with the cardiovascular sys-

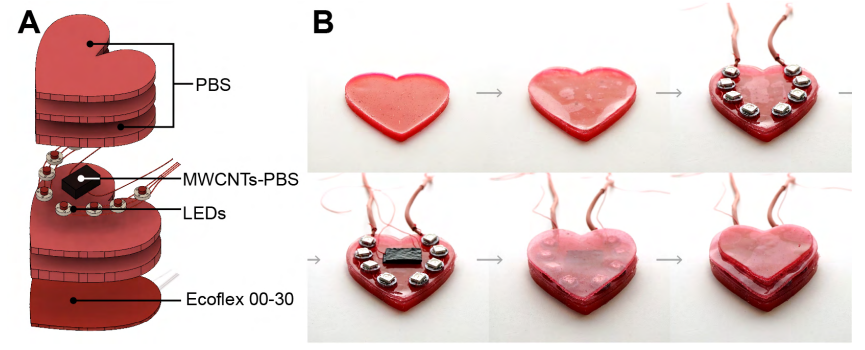

Figure 22. (A) A layered model of the healing heart. (B) Assembly process of the heart.

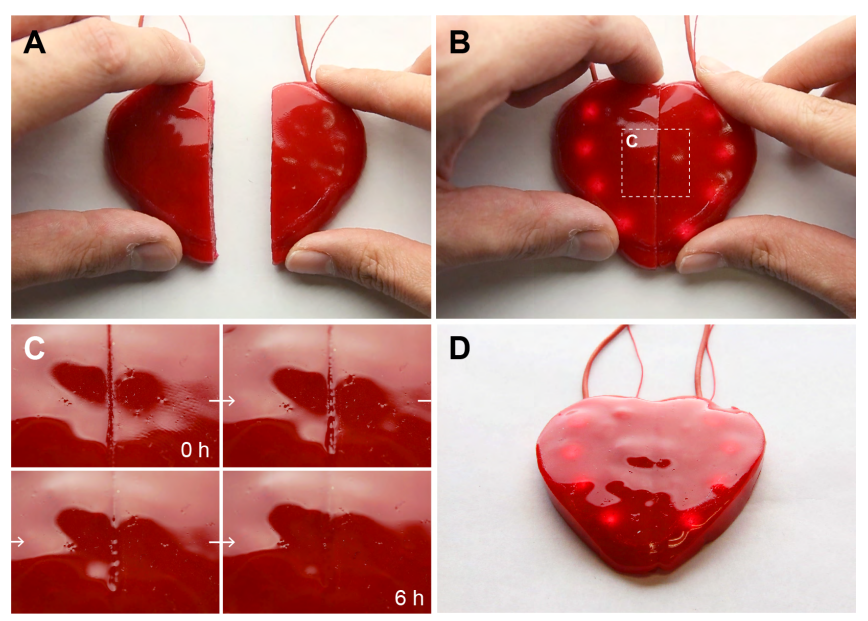

Figure 23. (A,B) The device can sense when the two halves are joined. (C,D) The heart can stick together after a few seconds and self-heals completely in $6 \mathrm{~h}$.

tem; a prior study suggests the maximum exposure of Baytubes MWCNTs, which we used in our study, to be $\sim 0.05$ $\mathrm{mg} / \mathrm{m}^{3}$ inside the lungs [39].

However, we observed that the MWCNTs powder used in our fabrication process forms small bundles of diameter $\sim 1 \mathrm{~mm}$ and thus are too heavy to become airborne. In addition, the strong interactions between MWCNTs and encapsulating PBS polymer matrix $[35,9]$ further prevent MWCNT bundles from releasing into the air even during cutting or tearing (see Figure S1 (e) in [35]). Thus our UIs do not expose the underlying cells to MWCNTs through skin contact. MWCNTs have already been used in consumer products including car tires, ship hulls, and water filters, showing that MWCNTs can be safely handled [10].

Nevertheless, we recommend following appropriate MWCNTs handling procedures described in the section of Preparation of Materials. We attached Consent Order Form of Baytubes MWCNTs and safety data sheet on general MWCNTs in Supplementary Information.

\section{Safety of PBS}

We also used PBS, which is made by reacting PDMS with boric acid. PDMS is used in biomedical applications due to its bio-inertness $[3,29]$. Boric acid is used in contact cleaning solutions, displaying low cytotoxicity [22]. PBS has even been used as a toy called Silly Putty [25]. 

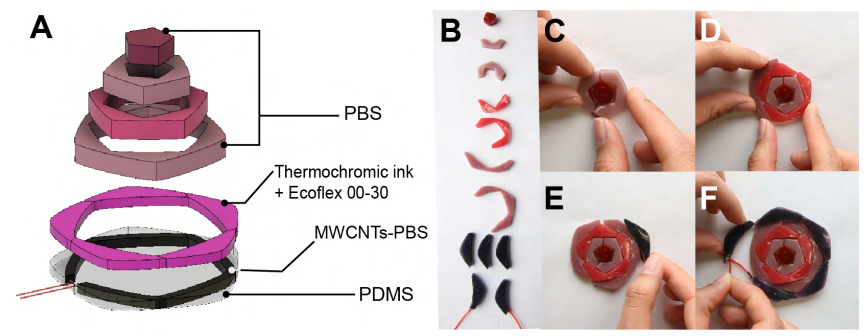

Figure 24. (A) A layered model of the fusing rose puzzle. (B-F) The assembly process.

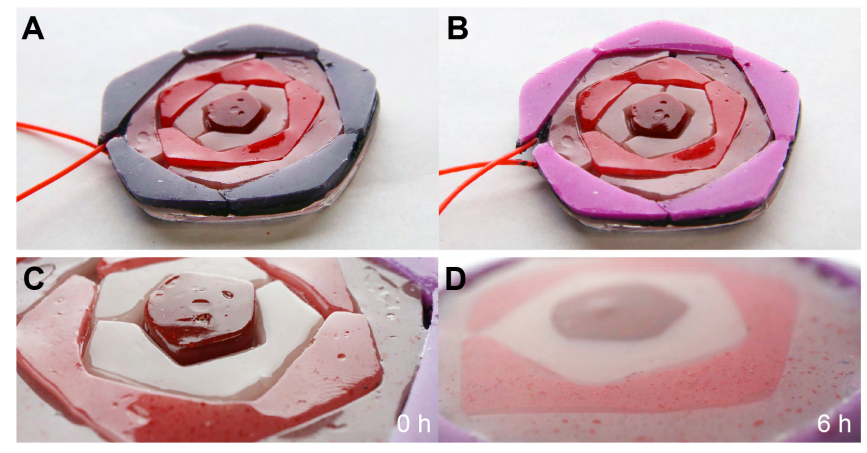

Figure 25. Fusing rose puzzle. (A,B) Right after joined, the thermochromic layer in the outer pieces was activated by the MWCNTs-PBS heater below. (C,D) In $6 \mathrm{~h}$, the puzzle fused into a unibody.

\section{Extreme heat applied to MWCNTs-PBS}

Although MWCNTs-PBS after preparation is safe for use, we should avoid applying heat over a few hundred degrees Celsius to it. The extreme heat could not only ruin the selfhealing property of PBS but also burn the PBS shell, making MWCNTs exposed to the air; this is the reason why we used automated cutting process with a drag knife, not laser cutting in which laser irradiation can cause overheating.

\section{LIMITATIONS AND FUTURE WORK}

\section{Ease of fabrication}

As explained above, our current fabrication method is based on molding, automated cutting, and stacking layers, which is time-consuming and restricted in terms of its design freedom, while laser cutting should be avoided. As an alternative way of fabrication, we expect a 3D printing process of PBS and MWCNTs-PBS can potentially replace the whole procedure as one of the future works.

\section{Different material choices for specific use}

While MWCNTs-PBS is easy to handle due to its elastic nature, PBS tends to be troublesome when we try to make a thin film (approx. $<1 \mathrm{~mm}$ ) with our current molding process. When we apply force to the thin PBS sheet to peel it off, the sheet can easily deform and sometimes result in fractured shape. Adding an appropriate amount of insulative particles into PBS and forming an insulative composite of PBS could help us enlarge the design freedom as well as make the process easier. The other option is to use a stiff self-healing material. Recently, intrinsic, autonomous, and mechanically robust selfhealing material was reported [57], which can be useful if we do not need the soft property of self-healing materials.
Also, our current method of pressure sensing has room for improvement. Due to the aspect ratio of MWCNTs particles, the resistance change responding to the pressure deformation is not significant, which causes a low $\mathrm{S} / \mathrm{N}$ ratio. It may be effective to replace the conductive material into graphite micro sheet that has more surface area than MWCNTs and will react more drastically to the deformation [5].

\section{"Ghosting" effect in the damage sensor}

Our current damage sensor is composed of a simple 2D sensor matrix, so in some cases, the sensor cannot correctly distinguish the place of cuts if we cut more than two places at the same time (so-called a ghosting effect, shown in Figure 19). In the future, a new structure should be explored to correctly detect multiple cuts on the sensor.

\section{Electrical disconnection due to the creeping of PBS}

As shown in Figure 8, PBS starts to creep and flow out if there is no constraint. In the prototype of the self-healing controller, we observed that wiring was pulled off by surrounding PBS and disconnected from the MWCNTs-PBS sensors. In order to prevent this issue, we need to either (1) anchor the wire to non-flowing material such as Ecoflex and MWCNTs-PBS or (2) constrain the PBS structure with the surrounding structure.

\section{CONCLUSIONS}

In this paper, we proposed Self-healing UI, a soft-bodied interface that can intrinsically self-heal its mechanical and electrical damages without external stimuli or glue. We proposed and implemented the hybrid devices of PBS, MWCNTs-PBS, and other components fabricated by the layer-by-layer structure. We also demonstrated the unique design space of self-healing materials through five applications. We strongly believe our Self-healing UI will enrich the field of HCI, as a new embodiment of material-driven shape-changing interfaces.

\section{ACKNOWLEDGMENTS}

This research was supported by the Carnegie Mellon University Manufacturing Futures Initiative (MFI) made possible by the Richard King Mellon Foundation; Carnegie Bosch Institute Research Awards; Commonwealth of Pennsylvania, Department of Community and Economic Development; and JST, ACT-I, Grant Number JP18070086, Japan. The authors would also like to thank Humphrey Yang and John Antanitis for technical assistance in the CNC drag knife; Guanyun Wang for the conceptual sketches and photographs; Michael Rivera and Danli Luo for technical advice; Gierad Laput for his help in laser cutting molds; Jack Forman for the support in the video shooting process; and the members of Morphing Matter Lab for discussion on the concept and the applications.

\section{REFERENCES}

[1] E. Acome, S. K. Mitchell, T. G. Morrissey, M. B. Emmett, C. Benjamin, M. King, M. Radakovitz, and C. Keplinger. 2018. Hydraulically amplified self-healing electrostatic actuators with muscle-like performance. Science 359, 6371 (2018), 61-65. D0I : http://dx. doi .org/10.1126/science. aao6139

[2] Jason Alexander, Anne Roudaut, Jürgen Steimle, Kasper Hornbæk, Miguel Bruns Alonso, Sean Follmer, and 
Timothy Merritt. 2018. Grand Challenges in Shape-Changing Interface Research. In Proceedings of the 2018 CHI Conference on Human Factors in Computing Systems (CHI '18). ACM, New York, NY, USA, Article 299, 14 pages. DOI : http://dx.doi.org/10.1145/3173574.3173873

[3] G. Bartalena, Y. Loosli, T. Zambelli, and J. G. Snedeker. 2012. Biomaterial surface modifications can dominate cell-substrate mechanics: the impact of PDMS plasma treatment on a quantitative assay of cell stiffness. Soft Matter 8 (2012), 673-681. Issue 3. DOI : http://dx.doi.org/10.1039/C1SM06250F

[4] Alberto Boem and Giovanni Maria Troiano. 2019. Non-Rigid HCI: A Review of Deformable Interfaces and Input. In Proceedings of the 2019 on Designing Interactive Systems Conference (DIS '19). ACM, New York, NY, USA, 885-906. DOI : http://dx.doi.org/10.1145/3322276.3322347

[5] Conor S. Boland, Umar Khan, Gavin Ryan, Sebastian Barwich, Romina Charifou, Andrew Harvey, Claudia Backes, Zheling Li, Mauro S. Ferreira, Matthias E. Möbius, Robert J. Young, and Jonathan N. Coleman. 2016. Sensitive electromechanical sensors using viscoelastic graphene-polymer nanocomposites. Science 354, 6317 (2016), 1257-1260. DOI :

http://dx.doi.org/10.1126/science . aag2879

[6] Stefano Burattini, Barnaby W. Greenland, Daniel Hermida Merino, Wengui Weng, Jonathan Seppala, Howard M. Colquhoun, Wayne Hayes, Michael E. Mackay, Ian W. Hamley, and Stuart J. Rowan. 2010. A Healable Supramolecular Polymer Blend Based on Aromatic $\pi$ - $\pi$ Stacking and Hydrogen-Bonding Interactions. Journal of the American Chemical Society 132, 34 (2010), 12051-12058. DOI : http://dx.doi.org/10.1021/ja104446r

[7] Mark Burnworth, Liming Tang, Justin R Kumpfer, Andrew J Duncan, Frederick L Beyer, Gina L Fiore, Stuart J Rowan, and Christoph Weder. 2011. Optically healable supramolecular polymers. Nature 472, 7343 (2011), 334. DOI : http://dx.doi.org/10.1038/nature09963

[8] Marcelo Coelho and Jamie Zigelbaum. 2011. Shape-changing Interfaces. Personal Ubiquitous Comput. 15, 2 (Feb. 2011), 161-173. DOI: http://dx.doi.org/10.1007/s00779-010-0311-y

[9] Jonathan N. Coleman, Umar Khan, Werner J. Blau, and Yurii K. Gun'ko. 2006. Small but strong: A review of the mechanical properties of carbon nanotube-polymer composites. Carbon 44, 9 (2006), 1624 - 1652. DOI : http://dx.doi.org/10.1016/j. carbon. 2006.02.038

[10] Michael F. L. De Volder, Sameh H. Tawfick, Ray H. Baughman, and A. John Hart. 2013. Carbon Nanotubes: Present and Future Commercial Applications. Science 339, 6119 (2013), 535-539. DOI :

http://dx.doi.org/10.1126/science. 1222453
[11] Eleonora D'Elia, Suelen Barg, Na Ni, Victoria G. Rocha, and Eduardo Saiz. 2015. Self-Healing Graphene-Based Composites with Sensing Capabilities. Advanced Materials 27, 32 (2015), 4788-4794. DOI : http://dx.doi.org/10.1002/adma. 201501653

[12] Sean Follmer, Daniel Leithinger, Alex Olwal, Akimitsu Hogge, and Hiroshi Ishii. 2013. inFORM: dynamic physical affordances and constraints through shape and object actuation. In Proceedings of the 26th Annual Symposium on User Interface Software and Technology (UIST '13). ACM, New York, NY, USA, 417-426. DOI : http://dx.doi.org/10.1145/2501988.2502032

[13] Antonio Gomes, Andrea Nesbitt, and Roel Vertegaal. 2013. MorePhone: A Study of Actuated Shape Deformations for Flexible Thin-film Smartphone Notifications. In Proceedings of the SIGCHI Conference on Human Factors in Computing Systems (CHI '13). ACM, New York, NY, USA, 583-592. DOI : http://dx.doi .org/10.1145/2470654.2470737

[14] Chris Harrison and Scott E. Hudson. 2009. Providing Dynamically Changeable Physical Buttons on a Visual Display. In Proceedings of the SIGCHI Conference on Human Factors in Computing Systems (CHI '09). ACM, New York, NY, USA, 299-308. DOI : http://dx.doi .org/10.1145/1518701.1518749

[15] Hiroshi Ishii, Dávid Lakatos, Leonardo Bonanni, and Jean-Baptiste Labrune. 2012. Radical Atoms: Beyond Tangible Bits, Toward Transformable Materials. interactions 19, 1 (Jan. 2012), 38-51. DOI : http://dx.doi.org/10.1145/2065327.2065337

[16] Henk M. Jonkers. 2007. Self Healing Concrete: A Biological Approach. In Self Healing Materials: An Alternative Approach to 20 Centuries of Materials Science, Sybrand van der Zwaag (Ed.). Springer Netherlands, Dordrecht, 195-204. DOI :

http://dx.doi .org/10.1007/978-1-4020-6250-6_9

[17] Hsin-Liu (Cindy) Kao, Christian Holz, Asta Roseway, Andres Calvo, and Chris Schmandt. 2016. DuoSkin: Rapidly Prototyping On-skin User Interfaces Using Skin-friendly Materials. In Proceedings of the 2016 ACM International Symposium on Wearable Computers (ISWC '16). ACM, New York, NY, USA, 16-23. DOI : http://dx.doi.org/10.1145/2971763.2971777

[18] Nicholas Kellaris, Vidyacharan Gopaluni Venkata, Garrett M. Smith, Shane K. Mitchell, and Christoph Keplinger. 2018. Peano-HASEL actuators: Muscle-mimetic, electrohydraulic transducers that linearly contract on activation. Science Robotics 3, 14 (2018). DOI :

http://dx.doi.org/10.1126/scirobotics . aar3276

[19] M. W. Keller, S. R. White, and N.âĂL'R. Sottos. 2007. A Self-Healing Poly(Dimethyl Siloxane) Elastomer. Advanced Functional Materials 17, 14 (2007), 2399-2404. DOI :

http://dx.doi.org/10.1002/adfm. 200700086 
[20] GW Lai, SJ Chang, JT Lee, H Liu, and CC Li. 2015. Conductive microcapsules for self-healing electric circuits. RSC Advances 5, 126 (2015), 104145-104148. DOI : http://dx.doi .org/10.1039/C5RA22021A

[21] Mathieu Le Goc, Lawrence H. Kim, Ali Parsaei, Jean-Daniel Fekete, Pierre Dragicevic, and Sean Follmer. 2016. Zooids: Building Blocks for Swarm User Interfaces. In Proceedings of the 29th Annual Symposium on User Interface Software and Technology (UIST '16). ACM, New York, NY, USA, 97-109. D0I : http://dx.doi.org/10.1145/2984511.2984547

[22] David M. Lehmann, Megan E. Cavet, and Mary E. Richardson. 2010. Nonclinical safety evaluation of boric acid and a novel borate-buffered contact lens multi-purpose solution, BiotrueâĎ́ multi-purpose solution. Contact Lens and Anterior Eye 33 (2010), S24 - S32. DOI :

http://dx.doi.org/10.1016/j.clae.2010.06.010

[23] Cheng-Hui Li, Chao Wang, Christoph Keplinger, Jing-Lin Zuo, Lihua Jin, Yang Sun, Peng Zheng, Yi Cao, Franziska Lissel, Christian Linder, and others. 2016. A highly stretchable autonomous self-healing elastomer. Nature chemistry 8, 6 (2016), 618. DOI : http://dx.doi.org/10.1038/nchem. 2492

[24] Xufeng Li, Dian Zhang, Kewei Xiang, and Guangsu Huang. 2014. Synthesis of polyborosiloxane and its reversible physical crosslinks. Rsc Advances 4, 62 (2014), 32894-32901. DOI : http://dx.doi.org/10.1039/C4RA01877J

[25] Zhen Liu, Stephen J. Picken, and Nicolaas A. M. Besseling. 2014. Polyborosiloxanes (PBSs), Synthetic Kinetics, and Characterization. Macromolecules 47, 14 (2014), 4531-4537. DOI : http://dx.doi.org/10.1021/ma500632f

[26] Qiuyu Lu, Chengpeng Mao, Liyuan Wang, and Haipeng Mi. 2016. LIME: LIquid MEtal Interfaces for Non-Rigid Interaction. In Proceedings of the 29th Annual Symposium on User Interface Software and Technology (UIST '16). ACM, New York, NY, USA, 449-452. DOI : http://dx.doi.org/10.1145/2984511.2984562

[27] James E Mark. 2009. Polymer data handbook. Oxford university press.

[28] Eric J Markvicka, Michael D Bartlett, Xiaonan Huang, and Carmel Majidi. 2018. An autonomously electrically self-healing liquid metal-elastomer composite for robust soft-matter robotics and electronics. Nature materials 17, 7 (2018), 618. DOI :

http://dx.doi.org/10.1038/s41563-018-0084-7

[29] Alvaro Mata, Aaron J. Fleischman, and Shuvo Roy. 2005. Characterization of Polydimethylsiloxane (PDMS) Properties for Biomedical Micro/Nanosystems. Biomedical Microdevices 7, 4 (01 Dec 2005), 281-293. DOI : http://dx.doi .org/10.1007/s10544-005-6070-2
[30] Ken Nakagaki, Artem Dementyev, Sean Follmer, Joseph A. Paradiso, and Hiroshi Ishii. 2016. ChainFORM: A Linear Integrated Modular Hardware System for Shape Changing Interfaces. In Proceedings of the 29th Annual Symposium on User Interface Software and Technology (UIST'16). ACM, New York, NY, USA, 87-96. DOI :

http://dx.doi.org/10.1145/2984511.2984587

[31] Ken Nakagaki, Sean Follmer, and Hiroshi Ishii. 2015. LineFORM: Actuated Curve Interfaces for Display, Interaction, and Constraint. In Proceedings of the 28th Annual ACM Symposium on User Interface Software \&\#38; Technology (UIST'15). ACM, New York, NY, USA, 333-339. DOI :

http://dx.doi.org/10.1145/2807442.2807452

[32] Ken Nakagaki, Luke Vink, Jared Counts, Daniel Windham, Daniel Leithinger, Sean Follmer, and Hiroshi Ishii. 2016. Materiable: Rendering Dynamic Material Properties in Response to Direct Physical Touch with Shape Changing Interfaces. In Proceedings of the 2016 CHI Conference on Human Factors in Computing Systems (CHI'16). ACM, New York, NY, USA, 2764-2772. DOI : http://dx.doi.org/10.1145/2858036.2858104

[33] Masaki Nakahata, Yoshinori Takashima, Hiroyasu Yamaguchi, and Akira Harada. 2011. Redox-responsive self-healing materials formed from host-guest polymers. Nature communications 2 (2011), 511. DOI : http://dx.doi.org/10.1038/ncomms 1521

[34] Aditya Shekhar Nittala, Anusha Withana, Narjes Pourjafarian, and Jürgen Steimle. 2018. Multi-Touch Skin: A Thin and Flexible Multi-Touch Sensor for On-Skin Input. In Proceedings of the $2018 \mathrm{CHI}$ Conference on Human Factors in Computing Systems (CHI '18). ACM, New York, NY, USA, Article 33, 12 pages. DOI: http://dx.doi.org/10.1145/3173574.3173607

[35] Youngseok Oh and Mohammad F. Islam. 2015. Preformed Nanoporous Carbon Nanotube Scaffold-Based Multifunctional Polymer Composites. ACS Nano 9, 4 (2015), 4103-4110. DOI : http://dx.doi.org/10.1021/acsnano. 5b00170

[36] Simon Olberding, Michael Wessely, and Jürgen Steimle. 2014. PrintScreen: Fabricating Highly Customizable Thin-film Touch-displays. In Proceedings of the 27th Annual ACM Symposium on User Interface Software and Technology (UIST '14). ACM, New York, NY, USA, 281-290. DOI :

http://dx.doi.org/10.1145/2642918.2647413

[37] Jifei Ou, Mélina Skouras, Nikolaos Vlavianos, Felix Heibeck, Chin-Yi Cheng, Jannik Peters, and Hiroshi Ishii. 2016. aeroMorph - Heat-sealing Inflatable Shape-change Materials for Interaction Design. In Proceedings of the 29th Annual Symposium on User Interface Software and Technology (UIST'16). ACM, New York, NY, USA, 121-132. DOI : http://dx.doi.org/10.1145/2984511.2984520 
[38] Allen Pan. 2014. Conductak. https://www .kickstarter. com/projects/1474433053/ conductak-stick-circuits-anywhere. (2014). Accessed: July 1, 2019.

[39] Jürgen Pauluhn. 2010. Multi-walled carbon nanotubes (Baytubes ${ }^{\circledR}$ ): Approach for derivation of occupational exposure limit. Regulatory Toxicology and Pharmacology 57, 1 (2010), 78 - 89. DOI : http://dx.doi.org/10.1016/j.yrtph.2009.12.012

[40] Ameya Phadke, Chao Zhang, Bedri Arman, Cheng-Chih Hsu, Raghunath A. Mashelkar, Ashish K. Lele, Michael J. Tauber, Gaurav Arya, and Shyni Varghese. 2012. Rapid self-healing hydrogels. Proceedings of the National Academy of Sciences 109, 12 (2012), 4383-4388. DOI : http://dx.doi.org/10.1073/pnas. 1201122109

[41] Isabel P. S. Qamar, Rainer Groh, David Holman, and Anne Roudaut. 2018. HCI Meets Material Science: A Literature Review of Morphing Materials for the Design of Shape-Changing Interfaces. In Proceedings of the 2018 CHI Conference on Human Factors in Computing Systems (CHI'18). ACM, New York, NY, USA, Article 374, 23 pages. DOI:

http://dx.doi.org/10.1145/3173574.3173948

[42] Majken K. Rasmussen, Esben W. Pedersen, Marianne G. Petersen, and Kasper Hornbæk. 2012. Shape-changing Interfaces: A Review of the Design Space and Open Research Questions. In Proceedings of the SIGCHI Conference on Human Factors in Computing Systems (CHI'12). ACM, New York, NY, USA, 735-744. DOI : http://dx.doi.org/10.1145/2207676.2207781

[43] Deepak Ranjan Sahoo, Timothy Neate, Yutaka Tokuda, Jennifer Pearson, Simon Robinson, Sriram Subramanian, and Matt Jones. 2018. Tangible Drops: A Visio-Tactile Display Using Actuated Liquid-Metal Droplets. In Proceedings of the 2018 CHI Conference on Human Factors in Computing Systems (CHI'18). ACM, New York, NY, USA, Article 177, 14 pages. DOI : http://dx.doi.org/10.1145/3173574.3173751

[44] Liyang Shi, Pinghui Ding, Yuzhi Wang, Yu Zhang, Dmitri Ossipov, and Jöns Hilborn. 2019. Self-Healing Polymeric Hydrogel Formed by Metal-Ligand Coordination Assembly: Design, Fabrication, and Biomedical Applications. Macromolecular Rapid Communications 40, 7 (2019), 1800837. DOI : http://dx.doi.org/10.1002/marc. 201800837

[45] Ryo Suzuki, Junichi Yamaoka, Daniel Leithinger, Tom Yeh, Mark D. Gross, Yoshihiro Kawahara, and Yasuaki Kakehi. 2018. Dynablock: Dynamic 3D Printing for Instant and Reconstructable Shape Formation. In Proceedings of the 31st Annual ACM Symposium on User Interface Software and Technology (UIST'18). ACM, New York, NY, USA, 99-111. DOI : http://dx.doi.org/10.1145/3242587. 3242659

[46] Zhenghai Tang, Yingjun Liu, Qingyi Huang, Jinshan Zhao, Baochun Guo, and Liqun Zhang. 2018. A real recycling loop of sulfur-cured rubber through transalkylation exchange of $\mathrm{C}-\mathrm{S}$ bonds. Green Chemistry 20, 24 (2018), 5454-5458. DOI : http://dx.doi.org/10.1039/C8GC02932F

[47] Seppe Terryn, Joost Brancart, Dirk Lefeber, Guy Van Assche, and Bram Vanderborght. 2017. Self-healing soft pneumatic robots. Science Robotics 2, 9 (2017), eaan4268. DOI :

http://dx.doi .org/10.1126/scirobotics. aan4268

[48] Udayan Umapathi, Patrick Shin, Ken Nakagaki, Daniel Leithinger, and Hiroshi Ishii. 2018. Programmable Droplets for Interaction. In Extended Abstracts of the 2018 CHI Conference on Human Factors in Computing Systems (CHI EA '18). ACM, New York, NY, USA, Article VS15, 1 pages. DOI:

http://dx.doi.org/10.1145/3170427.3186607

[49] Akira Wakita, Akito Nakano, and Nobuhiro Kobayashi. 2011. Programmable Blobs: A Rheologic Interface for Organic Shape Design. In Proceedings of the Fifth International Conference on Tangible, Embedded, and Embodied Interaction (TEI'11). ACM, New York, NY, USA, 273-276. DOI :

http://dx.doi.org/10.1145/1935701.1935760

[50] Guanyun Wang, Tingyu Cheng, Youngwook Do, Humphrey Yang, Ye Tao, Jianzhe Gu, Byoungkwon An, and Lining Yao. 2018. Printed Paper Actuator: A Low-cost Reversible Actuation and Sensing Method for Shape Changing Interfaces. In Proceedings of the 2018 CHI Conference on Human Factors in Computing Systems (CHI '18). ACM, New York, NY, USA, Article 569, 12 pages. DOI :

http://dx.doi.org/10.1145/3173574.3174143

[51] Yao Wang, Assaf Biderman, Ben Piper, Carlo Ratti, and Hiroshi Ishii. 2003. SandScape.

https://tangible.media.mit. edu/project/sandscape/. (2003). Accessed: July 1, 2019.

[52] Martin Weigel, Tong Lu, Gilles Bailly, Antti Oulasvirta, Carmel Majidi, and Jürgen Steimle. 2015. iSkin: Flexible, Stretchable and Visually Customizable On-Body Touch Sensors for Mobile Computing. In Proceedings of the 33rd Annual ACM Conference on Human Factors in Computing Systems (CHI '15). ACM, New York, NY, USA, 2991-3000. DOI :

http://dx.doi.org/10.1145/2702123.2702391

[53] Michael Wessely, Theophanis Tsandilas, and Wendy E. Mackay. 2016. Stretchis: Fabricating Highly Stretchable User Interfaces. In Proceedings of the 29th Annual Symposium on User Interface Software and Technology (UIST '16). ACM, New York, NY, USA, 697-704. DOI : http://dx.doi.org/10.1145/2984511.2984521

[54] Scott R. White, Nancy R. Sottos, Philippe H. Geubelle, Jeffrey S. Moore, Michael R. Kessler, S. R. Sriram, Eric N. Brown, and S. Viswanathan. 2001. Autonomic healing of polymer composites. Nature 409, 6822 (2001), 794. DOI : http://dx.doi.org/10.1038/35057232 
[55] Anusha Withana, Daniel Groeger, and Jürgen Steimle. 2018. Tacttoo: A Thin and Feel-Through Tattoo for On-Skin Tactile Output. In Proceedings of the 31st Annual ACM Symposium on User Interface Software and Technology (UIST '18). ACM, New York, NY, USA, 365-378. DOI :

http://dx.doi.org/10.1145/3242587.3242645

[56] Tongfei Wu and Biqiong Chen. 2016. Synthesis of Multiwalled Carbon Nanotube-Reinforced Polyborosiloxane Nanocomposites with Mechanically Adaptive and Self-Healing Capabilities for Flexible Conductors. ACS Applied Materials \& Interfaces 8, 36 (2016), 24071-24078. DOI :

http://dx.doi.org/10.1021/acsami.6b06137
[57] Yu Yanagisawa, Yiling Nan, Kou Okuro, and Takuzo Aida. 2018. Mechanically robust, readily repairable polymers via tailored noncovalent cross-linking. Science 359, 6371 (2018), 72-76. DOI :

http://dx.doi.org/10.1126/science. aam7588

[58] Lining Yao, Ryuma Niiyama, Jifei Ou, Sean Follmer, Clark Della Silva, and Hiroshi Ishii. 2013. PneUI: Pneumatically Actuated Soft Composite Materials for Shape Changing Interfaces. In Proceedings of the 26th Annual ACM Symposium on User Interface Software and Technology (UIST '13). ACM, New York, NY, USA, 13-22. DOI : http://dx.doi.org/10.1145/2501988.2502037 